\title{
A Review of Color Science in Dentistry: Colorimetry and Color Space
}

\author{
James C. Ragain* \\ Associate Professor, Department of Restorative Dentistry, University of Tennessee Health Sciences Center, College of Dentistry, Memphis, TN, USA
}

Received: May 29, 2015; Accepted: September 02, 2015; Published: January 29, 2016

*Corresponding author: Dr. James C. Ragain, Associate Professor, Department of Restorative Dentistry, College of Dentistry, University of Tennessee Health Sciences Center, Memphis, TN, USA, Tel: +901-448-1323; E-mail: jragain@uthsc.edu

\begin{abstract}
This article is the second in a series of review articles discussing the applications of color science in the clinical practice of dentistry. This installment discusses the colorimetry, which is the science and technology used to quantify and describe physically the human color perception. Theories of color space and clinically portable colorimetric devices developed for determining tooth shade in the dental practice is discussed.
\end{abstract}

\section{Introduction}

This article is the second in a series of articles that will review the fundamentals of color science as applied to the practice of dentistry. The first article discussed the basic human perception of color and reviewed the current accepted theory of human color perception. This article will discuss the principles colorimetry, color difference calculations, and portable, clinical colorimetric instruments. The third article will discuss the application of colorimetric devices in the dental office. Suggestions for determining shade selection in the dental practice will also be reviewed. The common theme for all three of the articles is that an understanding of the principles of color vision, color measurement, and the communication of color parameters will improve the restorative dentist's abilities to meet patient expectations for biomimetic dental restorations.

For many dental patients, restoring dental esthetics is of high importance in the course of their dental treatment. The blending of restorative materials with the teeth, producing a naturallooking appearance suitable to the patient and in which the dental restoratives cannot be seen, should be a major goal of the restorative dentist [1].

Restorative dental materials and prostheses matched to patient tooth color using contemporary clinical techniques generally provide results acceptable by most patients. However, shade guides with shade tabs are commonly used for shade selection and these techniques are very subjective, and often these techniques depend on numerous ambient factors and color vision acuity of the clinician. In the contemporary restorative dental practice colorimetric instrumentation can be used to choose a close color match between tooth structures and dental restorative materials.

\section{Colorimetry}

In the life sciences, such as physiology and psychology, color is dealt with in terms of the human visual process and sensation in the consciousness of the human as an optical observer [2]. The combined intensities of the wavelengths present in a beam of visible light reflected off of the surface of an object determine the property referred to as color. Visible light is a form of electromagnetic radiant energy in the wavelength range of approximately $380-780 \mathrm{~nm}$ which can be detected by the human eye [3]. An object must either emit light, or it must reflect or transmit incident light from an external source for it to be visible. It can be said that the perception of color is the result of a physiological response to a physical stimulus [4]. Colorimeters and spectrophotometers also process reflected light from the surface of an object, and these devices calculate the light flux data into color parameters that can be used to determine shade and calculate color differences [5]. These instruments can also be thought of as "observers." Portable models of these instruments can be used in clinical dentistry to determine tooth shade. The operation and capabilities of these instruments is better understood with a basic understanding of color theory and colorimetry.

Various methods have been devised for quantifying color and expressing color numerically, thereby making it possible to communicate colors more accurately. A "color space" is a specific organization of colors. Since "color space" is a specific term, identifying a particular combination of color model and mapping function, it tends to be used informally to identify a color model. In 1905, Munsell developed a method of expressing colors and color space utilizing paper color chips classified according to their hue, lightness, and chroma [5]. Hue is the attribute of color by which we distinguish red from green, blue, yellow, and so forth. Munsell called red, yellow, green, blue, and purple the principle hues. Adjacent colors in this series can be mixed to obtain a continuous variation from one color to another (Figure 1). This series can be arranged in a circle because it returns to a starting point. After inserting five intermediate hues for a total of ten hues, he used 


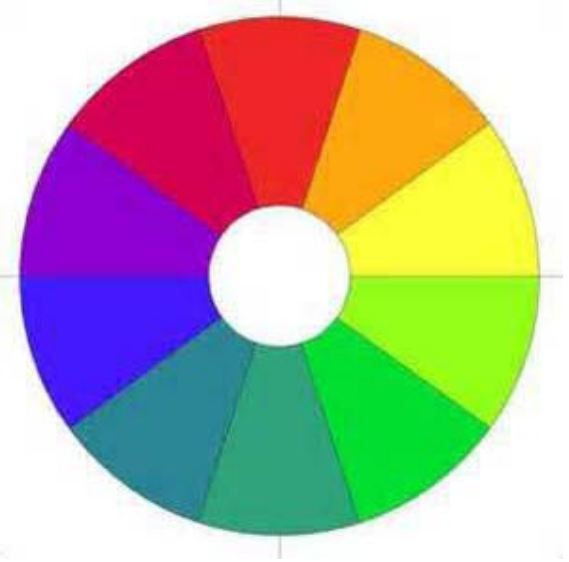

Figure 1: Munsell's hues.

initials as the symbols to designate the hue sectors (i.e., R, YR, Y, GY, G, BG, B, PB, P, and RP). The hue circle was divided into 100 steps with the zero point at the beginning of the red sector. Each step had an equal visual change in hue (Figure 2) [5].

Value indicates the lightness of the color, and the scale of value ranges from 0 for pure black to 10 for pure white. The whites, blacks, and grays are achromatic, having no hue. This lightness can be measured independently of hue. As the Figure 3 shows, lightness increases towards the top and decreases towards the bottom.

The degree of departure of a color from the neutral color of the same value is termed chroma. Colors of low chroma can be described as weak, while those of high chroma are strong or vivid. It is intended that the scaling of chroma be visually uniform. Chroma can be described as saturation levels of the hues. The units are arbitrary with the scale starting at 0 for neutral colors. There is no arbitrary end to the scale (Figure 3).

Munsell hue, value and chroma can be varied independently. In this way, all colors can be arranged according to the three attributes in a three-dimensional space (Figure 4). The neutral colors are placed along a vertical line, or neutral axis, with black at the bottom and white at the top. All grays on this axis are arranged between black and white. The hues are displayed at various angles around the neutral axis with the chroma scale is perpendicular to the axis and increasing outward. This threedimensional arrangement of colors is called the Munsell color space (Figure 5) [5]. This system was later updated to create the Munsell Renotation System in which any given color is expressed as a letter/number combination in terms of hue, value, and chroma as visually evaluated using Munsell Color Charts [6].

All colors lie within a specific region of Munsell color space called the "Munsell color solid". Hue is limited to one turn around the circle. The scale of value is limited on the lower end by pure black, which is as dark as a color can be, and on the top by pure white, which is as light as a color can be. For a given value, there is a limit to the chroma that is possible, even with theoretically ideal coloring agents. Real coloring agents, with less than ideal characteristics, impose further limitations on physical representations of the color solid. The Munsell Color Order System itself is applicable to all possible colors. The highest chroma yellow colors have rather high values, while the highest chroma blue colors have lower values.

These three color attributes can be put together to create the three-dimensional solid shown in Figure 6. Hues form the outer rim of the solid, with lightness as the center axis and saturation as
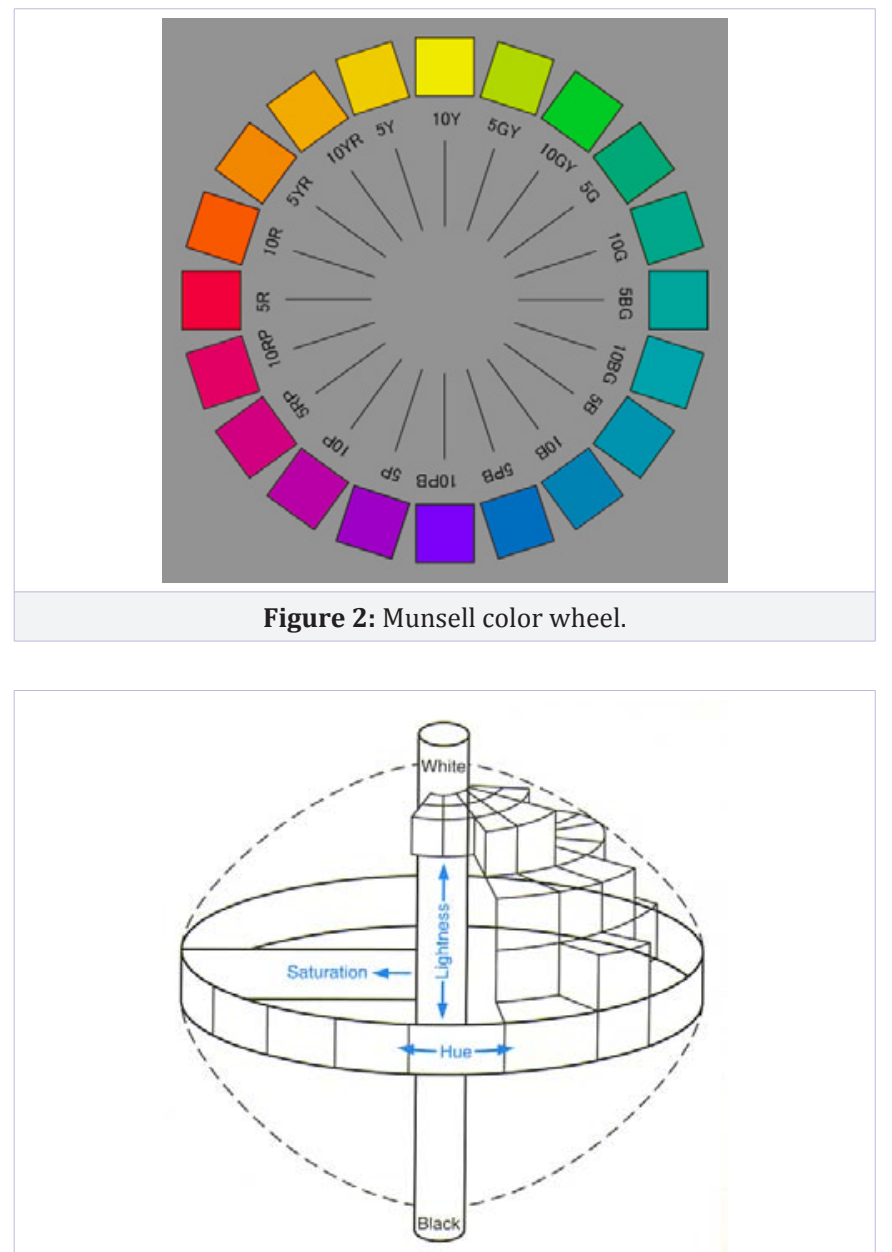

Figure 3: Munsell color wheel demonstrating arrangement of saturation, lightness, and hue.

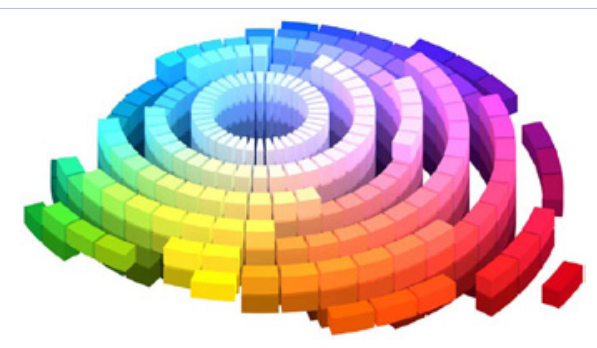

Figure 4: 3-D representation of Munsell renotations. 
the horizontal spokes. If the actual colors which exist in the world were distributed around the solid shown in Figure 5, the color solid shown in Figure 6 would be created. The shape of the color solid is somewhat complicated because the size of the steps for saturation are different for each hue and lightness, but the color solid helps us to better visualize the relationship between hue, lightness, and saturation.

In 1931, the Commission International de l'Éclairage (CIE), or International Commission on Illumination, defined the concept of the tristimulus values $\mathrm{X}, \mathrm{Y}$, and Z [5] based on the three-component theory of color vision. This theory states the eye possesses receptors for the three primary colors of red, green, and blue. All other colors are seen as a mixture of these primary colors [1]. $\mathrm{XYZ}$ tristimulus values and the associated Yxy color space form the foundation of present CIE color spaces. The concept for the $\mathrm{XYZ}$ tristimulus values is based on the three-component theory of color vision, which states that the eye possesses receptors for three primary colors (red, green, and blue) and that all colors are seen as mixtures of these three primary colors. The XYZ tristimulus values are calculated using these Standard Observer color matching functions [5].

The $\mathrm{L}^{*} \mathrm{a}^{*} \mathrm{~b}^{*}$ color space (also referred to as CIELAB) is presently one of the most popular spaces for measuring object color and is widely used in virtually all fields (Figure 7). It is one of the uniform color spaces defined by CIE in 1976 in order to reduce one of the major problems of the original Yxy space: that equal

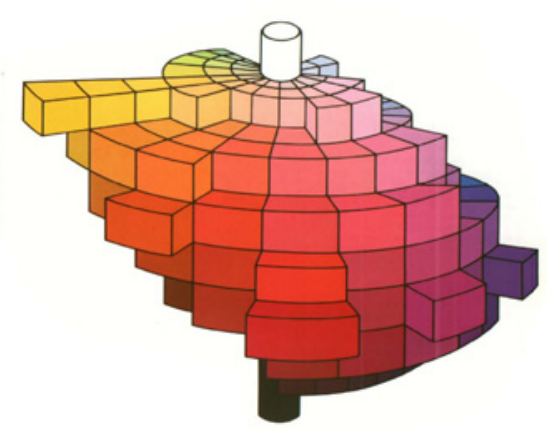

Figure 5: Munsell color space.

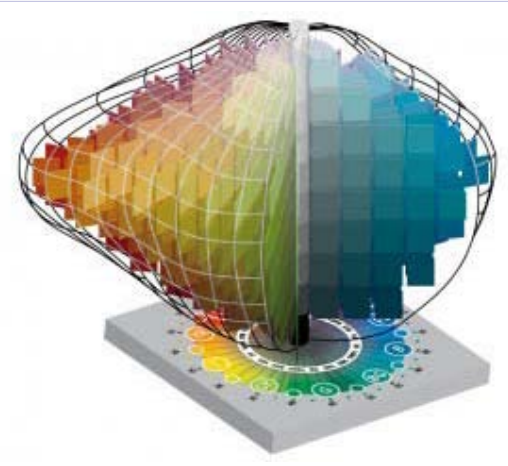

Figure 6: Munsell color solid.

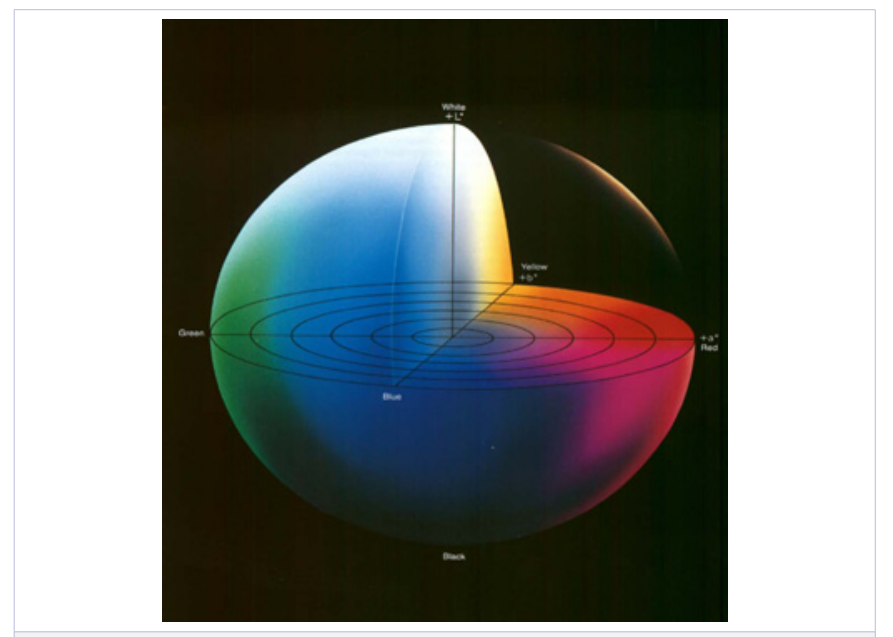

Figure 7: CIELAB Color Space.

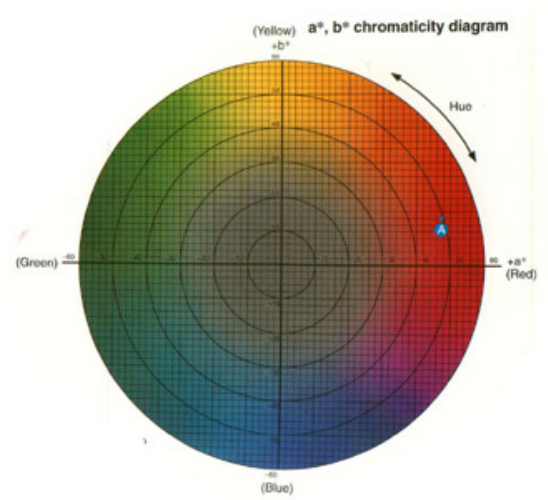

Figure 8: CIELAB a*, b* chromaticity diagram (Konica Minolta Precise Color Communication).

distances on the $\mathrm{x}, \mathrm{y}$ chromaticity diagram did not correspond to equal perceived color differences. In this space, $\mathrm{L}^{*}$ indicates lightness and $\mathrm{a}^{*}$ and $\mathrm{b}^{*}$ are the chromaticity coordinates. Figure 8 illustrates the $\mathrm{a}^{*}, \mathrm{~b}^{*}$ chromaticity diagram. In this diagram, the $a^{*}$ and $b^{*}$ indicate color directions: $+a^{*}$ is the red direction, $-a^{*}$ is the green direction, $+b^{*}$ is the yellow direction, and $-b^{*}$ is the blue direction. The center is achromatic; as the $a^{*}$ and $b^{*}$ values increase and the point moves out from the center, the saturation of the color increases.

Another color space paradigm, the $\mathrm{L}^{*} \mathrm{C}^{*} \mathrm{~h}$ color space, uses the same diagram as the $\mathrm{L}^{*} \mathrm{a}^{*} \mathrm{~b}^{*}$ color space, but uses cylindrical coordinates instead of rectangular coordinates. In this color space, $L^{*}$ indicates lightness and is the same as the $L^{*}$ of the $\mathrm{L}^{*} \mathrm{a}^{*} \mathrm{~b}^{*}$ color space, $\mathrm{C}^{*}$ is chroma, and $\mathrm{h}$ is the hue angle. The value of chroma $\mathrm{C}^{*}$ is 0 at the center and increases according to the distance from the center. Hue angle $h$ is defined as starting at the $+a^{*}$ axis and is expressed in degrees: 0 would be $+a^{*}$ (red), 90 would be $+\mathrm{b}^{*}$ (yellow), 180 would be -a* (green), and 270 would be $-b^{*}$ (blue).

The Hunter Lab color space was developed by R. S. Hunter as 
a more visually uniform color space than the CIE 1931 Yxy color space. Similar to the CIE L*a*b* space, it remains in use in various fields, including the paint industry of the US.

Minute color differences are difficult to deal with in industry anywhere that color is used. In the $\mathrm{L}^{*} \mathrm{a}^{*} \mathrm{~b}^{*}$ color space, color difference can be expressed as a single numerical value, $\Delta \mathrm{E}^{*} \mathrm{ab}$, which indicates the size of the color difference but not in what way the colors are different. $\Delta \mathrm{E}^{*} \mathrm{ab}$ is defined by the following equation:

$$
\Delta E_{a b} *=\sqrt{\left(\Delta L^{*}\right)^{2}+\left(\Delta a^{*}\right)^{2}+\left(\Delta b^{*}\right)^{2}}
$$

$\Delta \mathrm{E}_{\mathrm{ab}}^{*}=$ There are other color difference formulae in use today; however the CIELAB $\Delta \mathrm{E}_{\mathrm{ab}}$ is still the most widely used in color research in dentistry.

\section{Colorimeters and spectrophotometers}

The tristimulus method of colorimetry involves the use of an instrument call a colorimeter. This instrument measures the light reflected from an object using three sensors filtered to the same sensitivity, or color matching functions, ${ }^{\bar{x}}(\lambda), \bar{y}_{(\lambda) \text {, and }}$ $Z(\lambda)$ as the human eye. The colorimeter therefore calculates the tristimulus values $\mathrm{X}, \mathrm{Y}$, and $\mathrm{Z}$. The following formulas are used to determine the tristimulus values:

$$
\begin{aligned}
& X=K \int_{\lambda}^{\lambda} S(\lambda) \bar{x}(\lambda) R(\lambda) \Delta \lambda \\
& Y=K \int_{\lambda}^{\lambda} S(\lambda) \bar{y}((\lambda) R(\lambda) \Delta \lambda \\
& Z=K \int_{\lambda}^{\lambda} S(\lambda) \bar{z}(\lambda) R(\lambda) \Delta \lambda
\end{aligned}
$$

Where $\lambda$ is the wavelength of visible light (380-780), $\mathrm{S}(\lambda)$ is the relative spectral power of the illuminant, $\left(\bar{x}_{(\lambda)}, \bar{y}\right.$ $(\lambda)$, and $\bar{Z}(\lambda)$ are the color matching functions for the CIE $2^{\circ}$ observer $(, R(\lambda)$ is the spectral reflectance of the specimen, and $K$ is a normalizing constant and is derives as:

$$
K=100 / \int_{\Delta}^{\Delta} S(\lambda) \bar{y}(\lambda) \Delta \lambda
$$

This integration into the three color parameters allow for the metameristic effect that can occur between two materials with different spectral characteristics. Metamerism occurs when two materials of identical color under one spectral illuminant have different colors under another illuminant [7]. The tristimulus values can then be converted to the various color spaces.

Colorimeters are relatively small and portable [8]. Figure 9 is an example of a clinical colorimeter used in dentistry (Shofu

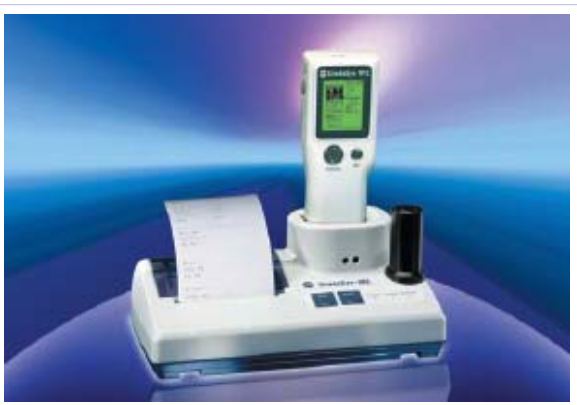

Figure 9: A dental colorimeter: Shofu ShadeEye NCC Tooth Colorimeter.

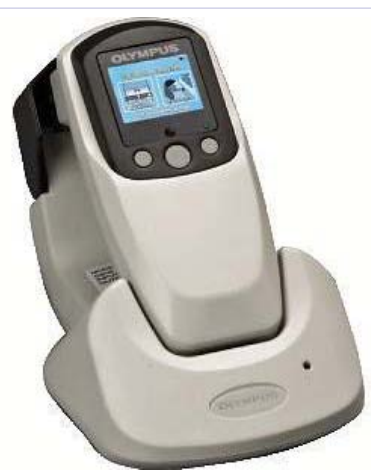

Figure 10: A dental spectrophotometer: Olympus America Crystal eye handheld dental spectrophotometer.

ShadeEye NCC Tooth Colorimeter ${ }^{\mathrm{TM}}$ ). Although the human eye cannot quantify colors accurately, it's a relatively simple task with a colorimeter. As we have seen previously, unlike the subjective expressions commonly used by people to describe colors verbally, colorimetersexpresscolors numerically according to international standards. By expressing colors in this way, it makes it possible for anyone to understand what color is being expressed. Further, a person's perception of a single color may change depending on the background or on the light source illuminating the color. Colorimeters have sensitivities corresponding to those of the human eye, but because they always take measurements using the same light source and illumination method, the measurement conditions will be the same, regardless of whether it's day or night, indoors or outdoors. This makes accurate measurements simple. Colorimeters can determine tristimulus values easily. The tristimulus colorimeter has features such as comparatively low price, compact size, superior mobility and simple operation. With a colorimeter, even minute color differences can be expressed numerically and easily understood. However, a colorimeter is not appropriate for complex color analysis such as metamerism and colorant strength [9].

When the color of objects is measured objects with a tristimulus colorimeter only numerical color data in various color spaces can be obtained. If a spectrophotometer is used for measurements, not only can the same types of numerical data be obtained, but the spectral reflectance graph for that color can also be obtained. Further, with its high-precision sensor 
and the inclusion of data for a variety of illuminant conditions, the spectrophotometer can provide higher accuracy than that obtainable with a tristimulus colorimeter [5]. An example of a dental clinical spectrophotometer is the Olympus America Crystaleye $^{\mathrm{TM}}$ handheld dental spectrophotometer (Figure 10).

A spectrophotometer has high precision and increased versatility. It is suitable for more complex color analysis because it can determine the spectral reflectance at each wavelength. However spectrophotometers can be more expensive than colorimeters. Always consider how accurately each color must be measured before selecting the type of instrument to use in a specific application [5].

\section{Conclusion}

The clinical use of colorimeters and spectrophotometers to determine the best match of dental material shade is on the increase in the practice of restorative dentistry. The third and last article in this series will review the clinical applications of colorimeters, spectrophotometers, and conventional shade guides.

\section{References}

1. Ragain JC. A Review of Color Science in Dentistry: The Process of Color
Vision. J Dent Oral Disord Ther. 2015;3(1):1-4.

2. MacAdams, David L. Color measurement, theme and variations. New York: Springer-Verlag; 1985.

3. Judd DB, Wyszecki G. Color in business, science, and industry. 3rd ed. New York: Wiley and Sons; 1975.

4. Kandel ER, Schwartz JH, Jessell TM, et al. Princilpes of neural science.5th ed. New York: McGraw-Hill Professional Publishing; 2012.

5. Ragain JC. Matching the Optical Properties of Direct Esthetic Dental Rstorative materials to Those of Human Enamel and Dentin. A dissertation for the partial fullfillment of the requirement for the $\mathrm{nf}$ degree Doctor of Philosophy in the Graduate School of The Ohio State University: Columbus, OH; 1998.

6. Hunt RWG. Measuring Colour. New York: Ellis Horwood; 1991.

7. Johnston WM. Color Measurment in dentistry. J of Dent. 2009; 37: e2e6.

8. Hunter RS, Harold RW. The measurement of appearance. 2nd ed. New York: Wiley and Sons; 1987.

9. Konica Minolta. Precise Color Communication. Online education. 2015. 